\title{
Climacturia, un sintoma a tener en cuenta tras prostatectomía radical
}

\author{
Loizaga Iriarte A, Paz Díaz-Romeral JL, Arciniega García JM, Arceo Santiago R, \\ Pérez Fernández A, Unda Urzaiz M.
}

\author{
Servicio de Urología. Hospital de Basurto. Bilbao
}

Actas Urol Esp. 2007;31(4):345-348

\section{RESUMEN}

CLIMACTURIA, UN SÍNTOMA A TENER EN CUENTA TRAS PROSTATECTOMÍA RADICAL

Introducción y objetivos: Los pacientes a los que se les ha realizado una prostatectomía radical describen disfunciones sexuales entre las que se encuentra el orgasmo asociado a incontinencia. Pretendemos valorar la incidencia y datos relacionados con este síntoma.

Métodos: Realizamos a 119 pacientes a los que se había realizado una prostatectomía radical, una encuesta telefónica dirigida a conocer la incidencia de climacturia. Pretendemos establecer la relación de éste síntoma con el grado y tipo de incontinencia, afectación a nivel de cuello vesical y apex, y la edad.

Resultados: La incidencia de climacturia fue del 20\%. La edad media de presentación del síntoma fue de 59 años, la misma que para los pacientes sin climacturia. Los pacientes con incontinencia y climacturia presentan fundamentalmente incontinencia de esfuerzo pero urgencia -incontinencia en mayor proporción (20\%) que los pacientes sin climacturia (5\%).

El paciente que tiene climacturia la presenta en forma de unas gotas en un $87 \%$ y siempre que llegan al orgasmo en un $62 \%$.

La influencia negativa del síntoma en la vida sexual del paciente y de su pareja solo se presentó en 2 casos (13\%).

Conclusiones: Proponemos el uso del mismo término en lengua castellana que en lengua inglesa. La edad y la afectación en cuello vesical y apex no influyen en la aparición de climacturia. En los pacientes con climacturia se asocia mas frecuentemente incontinencia de esfuerzo y el grado de incontinencia es mayor que en los que no la padecen.

Palabras clave: Disfunción eréctil. Prostatectomía radical. Incontinencia.

\section{ABSTRACT \\ CLIMACTURIA, A SYMPTOM TO TAKE INTO ACCOUNT AFTER RADICAL PROSTATECTOMY}

Introduction and objectives: Patients after radical prostatectomy describe sexual dysfunctions such as orgasm-associated incontinence also termed climacturia. Our aim is to analyse incidence and data in relation with this symptom.

Methods: 119 phone surveys carried out to patients after radical prostatectomy to Know about climacturia incidence. We are trying to show the relationship between this symptom and the degree and type of incontinence, affectation at bladder neck and apex, and the age.

Results: The climacturia incidence came to $20 \%$.The average age of the patients with this symptom was 59 years, the same as for those without it. The patients with both, incontinence and climacturia perform stress incontinence basically but urgency-incontinence to a greater extent (20\%) that those with no climacturia (5\%).

Most patients with climacturia suffer a light leakage (87\%) and a 62\% always associated to orgasm.

Negative effect of the symptom in the patient's and partner's sexual life appeared only in 2 cases (13\%).

Conclusions: We suggest the same term in Spanish than in English. Age and affectation of the bladder neck and apex do not have an effect on climacturia. Stress incontinence is more often related to patients with climacturia and the degree of incontinence is higher than in those without it. 
$\mathrm{L}$ os pacientes a los que se les ha realizado una prostatectomía radical describen disfunciones sexuales como la aneyaculación, la disfunción eréctil, enfermedad de Peyronie, la disminución del tamaño del pene y el orgasmo asociado a incontinencia $^{1}$. A esta última alteración se le ha denominado climacturia en estudios previos en lengua inglesa ${ }^{2}$ sin que hayamos encontrado ninguna referencia en la literatura en castellano.

Está aumentando el interés por este síntoma presentando trabajos en los congresos de la American Urological Association y de la Sexual Medicine of North America de los años 2005 y 2006 ,en $8^{\circ}$ Congreso de la European Society for Sexual Medicine y en Congreso de ISSM 2006.

\section{MÉTODOS}

Realizamos una encuesta telefónica a 119 pacientes a los que se había realizado una prostatectomía radical entre mayo de 2003 y mayo de 2005 dirigida a conocer la incidencia de climacturia. Pretendimos establecer la relación de éste síntoma con el grado y tipo de incontinencia , afectación a nivel de cuello vesical y ápex, y la edad.

El cuestionario incidía en la cantidad de pérdida de orina durante el orgasmo, la molestia que ello les suponía tanto a el paciente como a su pareja y su forma de evitarlo. El grado de incontinencia lo dividimos en minima (a grandes esfuerzos), leve ( 1 compresa al día), moderada (23 compresas al día), grave (mas de 3 compresas) y el tipo de incontinencia clínica en UrgenciaIncontinencia e Incontinencia de esfuerzo ${ }^{3}$.

La cuantificación de la climacturia la dividimos: en gotas, medio vaso aproximado y escape completo.

Criterios de inclusión: Pacientes a los que se les había realizado prostatectomía radical con conservación del cuello vesical por carcinoma de próstata localizado en nuestro hospital entre mayo 2003 y mayo 2005, con mas de 6 meses de seguimiento. Pacientes sexualmente activos.

Criterios de exclusión: Pacientes que no deseaban actividad sexual.

\section{RESULTADOS}

De los 75 pacientes que cumplian criterios de inclusión la incidencia de climacturia fue del 20\% (15 pacientes).
La edad media de presentación del síntoma fue de 59 años, la misma que para los pacientes sin climacturia.

Refiriéndonos al grado de incontinencia , los pacientes con climacturia presentaron en 2 casos, un grado de incontinencia grave; en 1, incontinencia moderada; en 3, incontinencia leve; en 4 incontinencia mínima y 5 casos no tenían ningún grado de pérdida de orina. Los pacientes sin climacturia presentaron en 1 caso incontinencia grave ; en 3, incontinencia moderada; en 14, incontinencia minima y en 32 no presentaron incontinencia. Sumamos en ambos grupos el grado de incontinencia leve, mínima y la no incontinencia , es decir que usaban 1 compresa al día o menos, para comprobar que el grado de incontinencia es bajo en los dos grupos $(80 \%$ en el grupo con climacturia y 93,3\% en el grupo sin ella) (Fig. 1) . Sin embargo en el grupo con climacturia el $20 \%$ tenían incontinencia moderada a grave frente al 6,6\% de los pacientes sin ella (Fig. 2).

Los pacientes que presentaban incontinencia, fundamentalmente era de esfuerzo aunque los pacientes con climacturia presentaban urgenciaincontinencia en un 20\% respecto al 5\% de los pacientes sin climacturia.

El 13\% (2/15) de los pacientes con climacturia y el 30\% (18/60) de los pacientes sin climacturia tenían afectación del ápex.

Ningún paciente tuvo afectación del cuello vesical en esta serie.

Los pacientes que referían climacturia la padecían rara vez, en un 13\%, a menudo en un $25 \%$ y siempre que llegaban al orgasmo en un 62\% (Fig. 3).

La mayoría de los pacientes describían la pérdida de orina durante el orgasmo como gotas $(87,5 \%)$; medio vaso solo 1 paciente y otro refería escape completo (Fig. 4).

La influencia negativa del sintoma en la vida sexual del paciente y de su pareja solo se presentó en 2 casos (13\%).

\section{CONCLUSIONES}

Proponemos el uso del mismo término en lengua castellana que en lengua inglesa.

La climacturia es un síntoma frecuente que si no se pregunta directamente los pacientes no lo describen. 


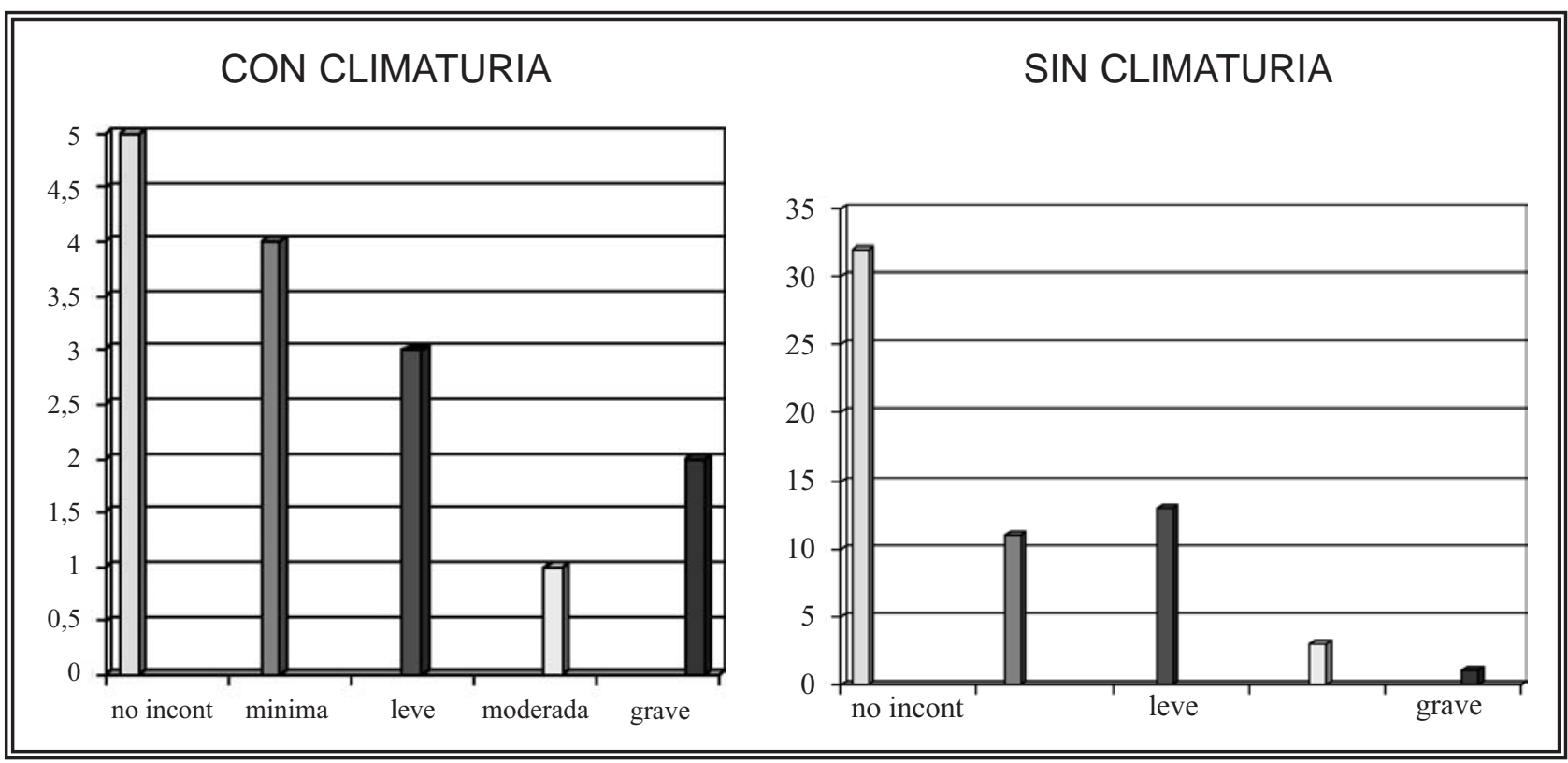

FIGURA 1. Resultados. Grado incontinencia.

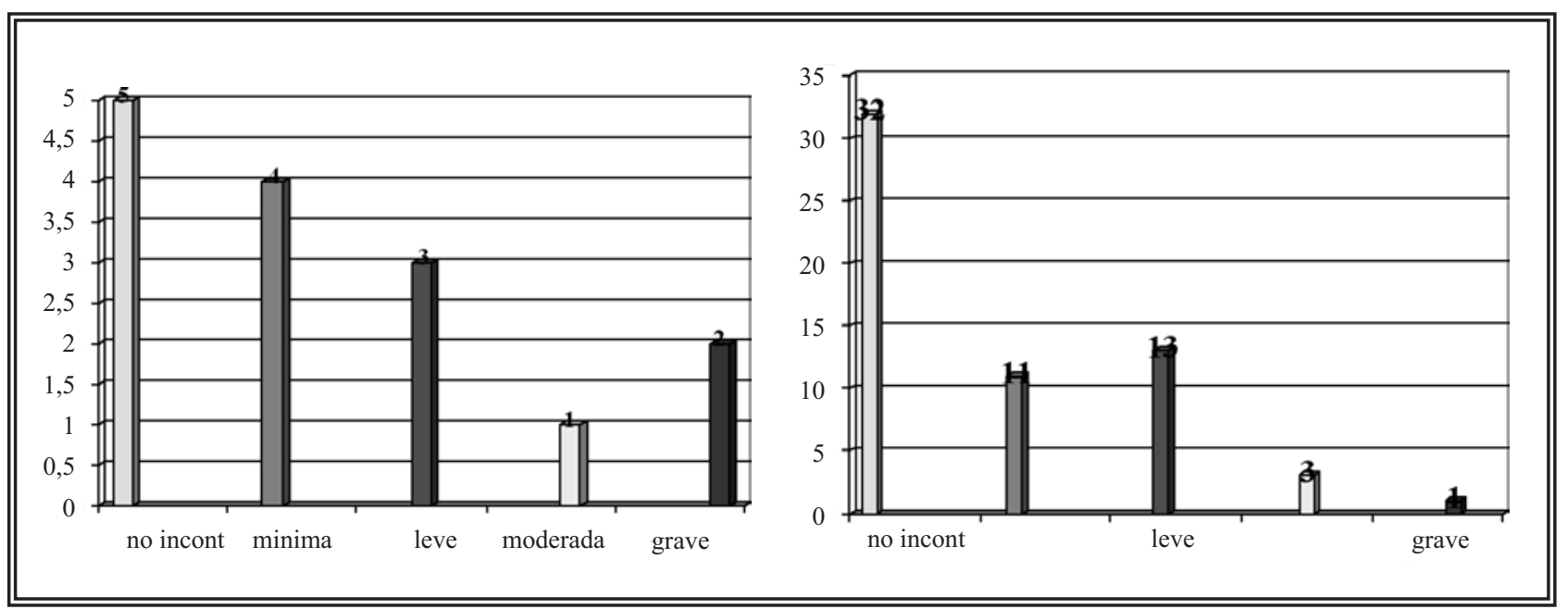

FIGURA 2. Resultados. Grado incontinencia.

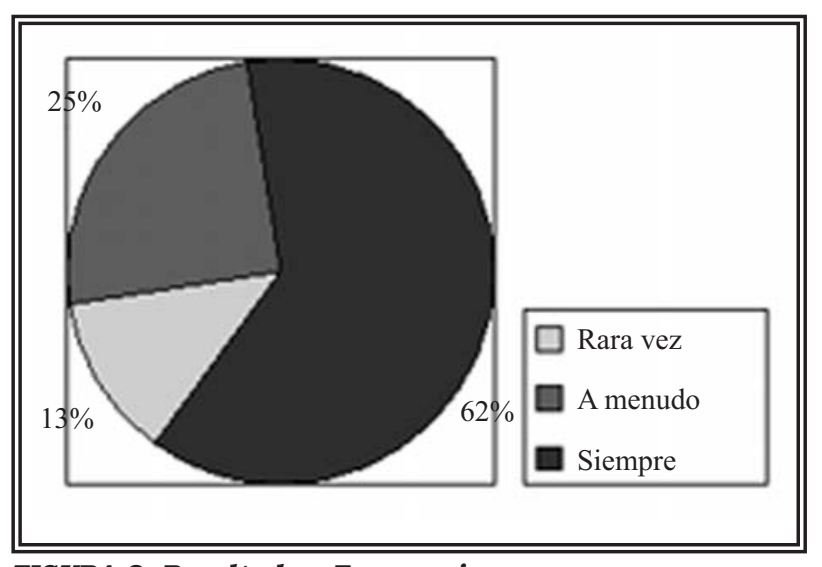

FIGURA 3. Resultados. Frecuencia.
La edad y los márgenes positivos en cuello vesical y ápex no influyen en la aparición de climacturia.

En los pacientes con climacturia se asocia mas frecuentemente incontinencia de esfuerzo y el grado de incontinencia es mayor que en los que no la padecen.

El paciente que tiene climacturia la presenta en forma de unas gotas en un $87 \%$ y siempre que llegan al orgasmo en un $62 \%$.

Es un sintoma que, por su frecuencia, debería formar parte de la información previa a la cirugía. Debiendo estar escrito en el consentimiento informado. 


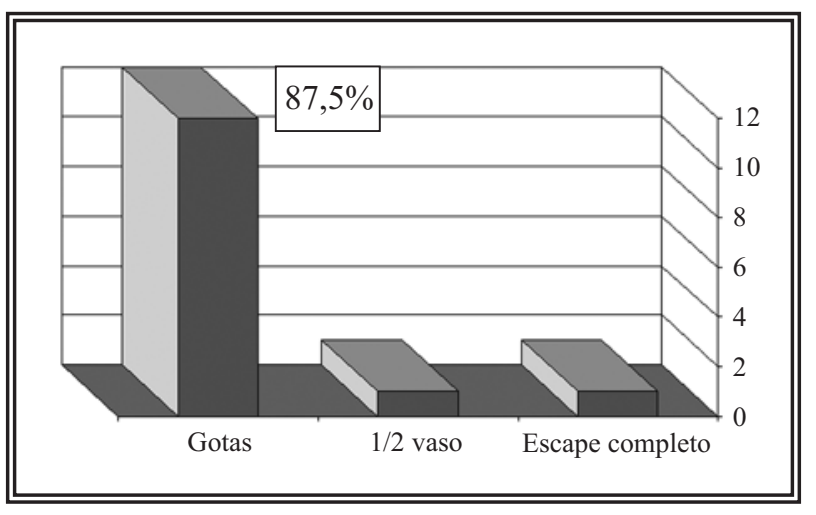

FIGURA 4. Resultados. Cuantificación de climacturia

Recomendamos dar las pautas para evitarla en la consulta postquirúrgica. La mejor manera de controlar el problema resulta tan sencilla como el vaciamiento vesical previo al acto sexual o el uso de preservativo.

\section{REFERENCIAS}

1. Augustin H, Pummer k, Daghger F, Habermann H, Primus G, Hubmer G. Patient self-reporting questionnaire on urological morbidity and bother after radical prostatectomy. Eur Urol. 2002Aug;42(2):112-117.

2. Lee J, Hersey K, Lee CT, Fleshner N. Climacturia following radical prostatectomy: prevalence and risk factors. J Urol. 2006; 176(6Pt1):2562-2565. Discussion 2565.

3. Lepor H, Kaci L. The impact of open radical retropubic prostatectomy on continence and lower urinary tract symptoms. A prospective assessment using validated selfadministered outcome instruments. J Urol. 2004 Mat;171 (3): 1216-1219.
Correspondencia autora: Dra. A. Loizaga Iriarte Servicio de Urología .Hospital de Basurto. Montevideo 18. 48013 Bilbao

Tel.: 944006160

E-mail: ana.loizagairiarte@osakidetza.net

Información artículo: Original - Cáncer de próstata

Trabajo recibido: enero 2007

Trabajo aceptado: febrero 2007 